\title{
TWENTY-FIVE YEARS OF THE SEARCH FOR EXTRATERRESTRIAL COMMUNICATIONS
}

\author{
P. Morrison \\ Department of Physics \\ Massachusetts Institute of Technology \\ Cambridge, MA 02139 \\ U.S.A.
}

\begin{abstract}
On this silver anniversary of the first published proposal for a search of the skies to seek communications over interstellar distances, I present a rather personal account of the origins of the proposal, and an appraisal of the new and hopeful status of the search worldwide.
\end{abstract}

\section{BEGINNINGS}

Our initial paper (Cocconi and Morrison 1959) was begun in Ithaca and completed in Geneva, at the brand-new CERN where Cocconi had gone as summer came that year, and where he has lived and worked ever since. We felt it an original if rather eccentric contribution; Giuseppe wrote privately to Sir Bernard Lovell to suggest the use of the big Jodrell Bank dish in the effort, but Lovell was not persuaded. I sent our paper along to Professor P.M.S. Blackett to seek his good offices in publication. A good friend and a man who liked novelty, he acted promptly and the paper soon appeared. Whatever the astronomers thought of the suggestion, the popular press was much taken with it. I spent that year on leave in a round-the-world set of visits, and the press followed me for comments from London to Rehovoth, Bombay, Kyoto, Denver and home again. Everyone surely knows that Frank Drake, quite independently of our paper, was at NRAO making ready in Green Bank the first real radio search, quite minimal by today's standards, but enough to show what we did not then know: not all main-sequence dwarfs are powerful sources of $\mathrm{CW}$ signals at 21 centimeters!

Both Cocconi and I were caught up in the larger currents of physics during the decades before. He is a wonderfully reflective experimenter, I a somewhat experimentally-oriented theorist, both moving out of the pre-war days of nuclear physics, into what had passed 
for particle physics in those naive years: cosmic rays. (For me wartime had meant the engineering of chain reactions fast and slow; Giuseppe recalled air raids more than wartime projects.) Mesons of more than one sort, then the V-particles and other enigmas were all newly found in cosmic rays. But in the early fifties the cosmic-ray physicists began to see that the new particle physics would be machine-made. The new beams of the engineers were much more useful than the celestial accelerator could provide, in every way save that of energy per particle.

But it became clearer and clearer in the fifties that cosmic rays would no longer dominate the experimental study of fundamental particles. Yet cosmic rays raised other questions that could not be be answered within the accelerator lab. Where did cosmic rays come from? How did they travel? What made them vary year by year, and sometimes even change intensity an order of magnitude within a few hours? These were questions of astronomy, in the solar system and beyond. The interests of many cosmic-ray physicists shifted from the gamut of new particles to the site and nature of new astronomical processes. We had known for a few years that the primary cosmic rays were some sort of a sample of cosmic matter as a whole.

High-energy astronomy became explicit and fascinating for me in an unforgettable meeting of cosmic-ray physicists at Guanajuato in Mexico about 1955. We then learned at first-hand the new Soviet results on polarized optical light from the Crab Nebula. Not so long before we had stared in wonder at the bright blue glow of the optical synchrotron radiation (prudently, we looked at it only in a mirror) produced by the new fast-electron beam in the Cornell synchrotron. It was a kind of light, I recall saying, quite new under the sun, for it arose from transitions in a magnetic fleld, and had no need for Coulomb interaction. Now we realized that it had lighted explosive stars and even solar flares for a long time. As below, so above: hardly a cosmic novelty.

In 1958 that line of thought led me to publish an account of the originating processes and possible importance of gamma-rays in astronomical contexts. I was far too optimistic; it took the experimenters a dozen years and more to develop the art of measurement. But there could be astronomical gamma-ray sources just as we were familiar with gamma rays from our synchrotron at Cornell. Optical synchrotron light was only a minor by-product of a strong fast-electron source, the possible source of gamma-rays. As below, so above?

Early in $1959 \mathrm{my}$ ingenious friend Cocconi came to me to speculate that perhaps gamma-ray beams could be found in space, generated by other beings just as the Cornell synchrotron had produced them. My paper had made clear that the rays could penetrate the Galaxy. That was the beginning of the new paper, in which we systematically examined the entire EM spectrum for a signalling optimum. Still tyros at radio astronomy, we prepared the letter to Nature as best we could; microwave radio easily led all the other bands in our simplified signal-to-noise study.

\section{THE DIMENSIONS OF SEARCH}

The search for signals, whether deliberate or mere accidental leakage, is certainly multi-dimensional. The obvious dimensions of direction in space, time, particle type (which 
for EM signals reduces to two polarizations), and signal intensity all require analysis, but I shall discuss mainly the frequency-time plane.

The overall argument in favor of microwaves rests of course on the spectrum of the natural noise in space. Taken with the strong stellar sources of optical and infrared light, and the quantum fluctuations of any photon-borne signal, the studies agree that somewhere between 1 and $10 \mathrm{GHz}$ is the most suitable frequency domain for energy-efficient signals at interstellar distance. Human technology has already acquired the means of interstellar signalling in these octaves, and only there. Let us accept this time-tried result, always only tentatively.

The next fixed point is the fundamental relation between band width and signal duration, $\Delta \nu \Delta t=1$. The search for sharp pulses, with small $\Delta t$, is one attractive limit, early pursued in the USSR (Troitskii et al. 1979). Plainly one goes to a pulse width such that $\Delta \nu / \nu$ is not very small, say only one or a few powers of ten below unity. For the microwave domain, that means pulses in the sub-microsecond range. (A physical lower limit to pulse length is in fractional nanoseconds.) The receivers now can be broad-band; a search to match receiver time to the pulse time is really implied, parallel to the ordinary radio search over frequency, but a pulse might be quite powerful and need little time averaging. Such was the early Soviet search mentioned; what it found was instructive, if rather to be expected. Plenty of phenomena much simpler than radio astronomers are able to generate sharp pulses. Geophysical pulses, their exact origin not known (at least to me), showed up in receivers spaced apart ten thousand kilometers. The sharp pulse, a limiting type of signal quite useful by mutual design, does not appear easy to employ between partners with no chance to prearrange the situation. They are too commonplace.

The other limit is clear: narrow-band carriers, with $\Delta \nu$ small. Then $\Delta t$ is large; these signals can vary only slowly. Most familiar radio circuits are of that kind, to be sure often for the frugal utilization of a crowded spectrum. It looks as though energy savings would be greater the smaller the bandwidth used. Moreover, we never encounter any natural celestial signal at all that could be called narrow-band in engineering terms. Since the first signal acquisition, if not the transmission of much intelligence, could be made on very narrow bands indeed, it is very important to recognize, as Drake and Helou 1976 first did, that in the Galaxy a physical lower limit to bandwidth is set by the dispersion imposed by plasma drifts in interstellar space. There is no gain in going below about a hundredth of a $\mathrm{Hz}$ for $\Delta \nu$. It is a search for this wonderful limiting case, the extreme narrow-band approach, standard for human signals, unknown in the skies, even in masers, which is at present realized by Paul Horowitz of Harvard (well-described in this volume).

There is much more choice for signal structure than the two extremes of narrow-band CW and wide-band sharp pulse; the whole domain invites eventual study. But the limiting cases are visibly attractive for our search.

\section{INSPIRED (?) GUESSES}

Systematic search over all the combinations of parameters that define the multidimensional possibilities is daunting. From the first, anyone looking at the problem is 
tempted to make an inspired guess, to invoke a piece of magic, that is, an attractive assumption that all at once sets a preferred value of some parameter, noticing a clue or hunch that may appeal to all reasonable searchers, at either end of the hoped-for signal link. For direction, one invokes evolutionary symmetry; we look for stars more or less like our own. There are certainly plenty of those. Or perhaps one looks at groups of stars all together, a cluster. Most likely one looks in all possible directions using a finite antenna beam. For frequency, even in our first paper the 21-centimeter line, the most abundant photon in the universe, stood out as a tempting spectral landmark. That choice still appeals to most of the radio astronomers who have attended to the problem. Plainly this is no objective matter; one can only argue plausibility, and then depend upon hope. Many alternatives will be discussed even in the present meeting.

It is of some value to recall that during the first twenty years of search we of earth, investigators in nine countries, tried three dozen pioneering searches. Nearly all used the broad microwave band. (An up-to-date review of every previous and on-going search is an important paper in this volume, presented by Jill Tarter.) Meanwhile our farthest space probe has sped out from the sun perhaps 25 or 30 astronomical units, almost ludicrously short of the stars. If that rudimentary experience is any guide, it confirms the old judgment that electromagnetic search is far easier: easily-created bosons handily win over conserved and costly fermions. (The almost uncoupled neutrinos have few supporters.)

\section{CHANGE}

Since 1959 receivers have gotten better, and there are fine new radio dishes. None is larger than the dish at Arecibo, in design way back then. Nor do the receiver improvements amount to an order of magnitude. What has changed by an unforeseeable factor, many orders of magnitude, is computing power, made possible by microelectronics. It would have been the state of the art to invoke a receiver capable of integrating the signal in 1024 channels at once. Right now Horowitz has two multi-channel receivers in everyday reliable operation integrating over a tenth of a megachannel all at once, and multi-channel devices, to be described here in detail, are under construction that listen to ten megachannels at a time. The use of that informal unit, the megachannel, makes clearer than anything else I can say what a new epoch has arrived. The search over wavebands has been empowered beyond the dreams of 1959.

A second novelty has come from astronomy. It is nothing so general as the rise of microchips, but it offers a certain elegance. It bears on the old guess that the 21-centimeter line is the place to look.

The trouble with a guessed frequency is of course that the natural line is not only a place of high noise power, but is also poorly-defined in frequency position. The natural line is megahertz wide; searching such a band in tiny nibbles of a hundredth of a hertz for every direction in space is a chore, even for megachannel receivers. Can we narrow our guess?

The natural width is a consequence of Doppler shifts; gases in space always move. Transmitters and receivers move too, so the signal can be given a precisely-assigned fre- 
quency only if there is some natural frame of reference that stays at rest, known both to transmitter and receiver. There is no place in the Galaxy that fits the bill, save its center. Since we move more or less transverse to that center, we might use it. But there is now known a much grander rest frame, that of the thermal background radiation, the mean motion of all the matter in the universe. Surely we can expect that all observers know that frame; we ourselves know it fairly well now. In a few years we can expect to fix it within a part in ten thousand or so. That implies the opportunity to tune to a 21-centimeter signal within a matter of a hundred kilohertz, a search within ten million minimal-width channels. We will soon be able to do that in one integration time. Of course we must correct away all the motions of sun and earth, as the transmitter must do for its part. But the microwave background, observable everywhere, renders the procedure entirely a matter of care, independently and mutually practical at both ends of the link.

That seems the happiest of hopeful guesses to me: the most abundant photon of all, frequency fixed in the grandest of all rest frames, agreement a matter of independent measurement. The scheme works for any signal path within the Local Group of galaxies. If we ever extend our search across tens of millions of light-years to more distant galaxies, the same frame offers other possibilities for a frequency choice that can correct for the velocity change with time that we call the red shift; that has been proposed by J.R. Gott of Princeton (Gott 1979).

\section{CRITICISM}

Most, though not all, of those who have looked into the matter agree that it is less useful now to estimate the chances of success from various a priori points of view than it is to mount as powerful a search as possible. Certainly that is the lesson from astronomy as a whole. All the same, it is useful to engage two particularly well-expressed criticisms of present efforts.

The most direct is a position that argues that no signal is likely to exist (Bates 1978) because no conceivable society would signal into the unknown for a time long enough to have a chance of an answer, perhaps thousands of years or even more. How could any government, Professor Bates asks, ever be concerned about posterity to such an extent? One important rejoinder is this: the notion of protracted search is not a necessary part of the decision. It is enough for each generation to begin the task anew, or by improvement upon the past. There is no requirement for absolute continuity; the game is a probabilistic one. Only the duty cycle really matters, how much of total time is spent in a signalling mode. Each new technique, each new insight, may bring a wave of activity. If the wave does not succeed, very likely it will die out, until the next impetus. That is the lesson of our own science; the Greek philosophers were the forerunners of the particle physicists of today. The enterprise of the ultimate analysis of matter is older than any government, university, or laboratory. If it dwindles for a while, it can grow again. Only a final end to all investigation can kill it. Without making any claim to certainty, I will at least argue the possibility of such a course for the searchers after signals from afar, whether in the cheap receiving mode or in the more advanced state of deliberate transmission. Leakage detection is also a minor possibility. 
Much better-known is a stance I have called Malthusian. It rests on the idea of the exponential improvement in all technology. That must entail, the proponents say, an eventual physical tour by crews or by mere automata, of the whole Galaxy. Using various estimates, all of which really exploit mainly the unbounded quality of an exponential rise, these critics say that we must be the first ever to think of beings afar. Otherwise they would have been here already; and where are they? This argument is modified to include deliberate concealment, the "zoo" idea, in which we humans have been preserved as unwitting pristine specimens, and more. All of this is interesting, but it shares the defect of Malthus. In the real world there are no unlimited exponentials. Something limits every growth. We are surely finite beings, and it is likely that we will remain finite forever, unless in finite time we disappear into zero. I do not know what the upper bound of our grasp can be; I suspect it will fall short of making a rose garden out of the Galaxy. With that finiteness the power of the argument fades; it all becomes a discussion over the values of limiting parameters that none of us know.

No, the best means to seek the unknown is by experiment, absent a tested theory able to exclude the result we seek-and even then, be careful about the truncation of experience. Consult any textbook for examples.

\section{MIXED STRATEGY}

The task before us is to organize the best search we can, and to improve it steadily. That certainly means that we do not dogmatically optimize all that we do according to the most popular assumptions. There is a place in every particular scheme of search for a logical design optimizing the search that follows from the assumptions.That is good engineering. But where we lack any real theory of what we seek we must take a more modest and more resilient stand. We ought to search with some effort in all the modes and under all the assumptions that we can convince ourselves remain possible. We can well spend most effort on the most widely-held views, but it is a mistake to close tight every other door. Unlikely strategies deserve, not equal time, but time and effort roughly proportioned to their judged probability. It would be wrong to bet everything on 21-centimeter signals from solar-type stars, even though that appeals most to me and to many others. For if the best-supported view turns out to be wrong, even if that is unlikely, and one has done nothing else, failure is very much more probable than with a prudently mixed, or hedged, strategy. Gamblers who do not hedge are not reasonable. Thus the NASA study wisely includes both narrowand wideband microwave search, and looks both at random star fields and at nearby target stars like the sun. There is and will be room for every plausible stratagem; let a.hundred schools contend, the least accepted perhaps contending on a shoestring. Overall strategy can be controlled over the long run, as always, only by the engaged community as a whole. Fads and follies will occur, but they will run their course. 


\section{IN CONCLUSION}

This meeting and its record here say much of value about these large questions. Unlike most of science, this topic extends beyond the test of a well-framed hypothesis; here we try to test an entire view of the world, incomplete and vulnerable in a thousand ways. That has a proud name in the history of thought as well; it is called exploration. We are scientists and engineers from a dozen backgrounds joined in the early ingenuous stages of a daring exploration, become real only during recent years.

It is a voyage whose end we do not know, like that of science itself. We seek a call, not from person-to-person, but from culture to culture, a call whose content we cannot well foresee. It is only the first step in opening such a channel, the process that communication specialists call the acquisition of a signal, that is now the focus of our work. Let us try as best we can; there is no other way.

\section{REFERENCES}

Bates, D. R. 1978, Astrophys. and Sp. Sci., 55, 7-13.

Cocconi, G. and Morrison, P. 1959, Nature, 184, 844-846.

Drake, F. D. and Helou, G. 1976. Report 76, National Astronomy and Ionosphere Center (Cornell University, Ithaca, New York).

Gott, J. R. 1982, Cosmology and Life in the Universe, in Extraterrestrials Where are They?, Hart, M. H. and Zuckerman, B. eds. (Pergamon: New York), 122134.

Troitskii, V. S., Starodubstev, A. M., and Bondar, L. N. 1979, 'Search for Radio Emissions from Extraterrestrial Civilizations,' Acta Astronautica, 6, 81-94. 